Minireview

\title{
Ironies in photosynthetic electron transport: a personal perspective
}

\author{
William A. Cramer \\ Department of Biological Sciences, Lilly Hall of Life Sciences, 915 West State Street, Purdue University, \\ West Lafayette, IN 47906-2054, USA (e-mail: wac@bilbo.bio.purdue.edu; fax: +1-765-496-1189)
}

Received 24 October 2003; accepted in revised form 29 November 2003

Key words: Jerry Babcock, Derek Bendall, Mick Black, L. Bogorad, Herbert Böhme, Warren L. Butler, Tony Crofts, crystal structure, cytochrome, cytochrome $b_{6} f$ complex, electron transfer, Mike Everly, Paul Furbacher, Mark Girvin, John Gray, Reinhold Herrmann, Peter Horton, Deru Huang, Anne and Pierre Joliot, Bessel Kok, Genji Kurisu, Dick Malkin, Daniel Picot, plastoquinone, Misha Ponamarev, Jean-Luc Popot, proton transfer, Rieske iron-sulfur protein, Janet Smith, Glenda Soriano, David Stroebel, Gun-Sik Tae, Achim Trebst, Olivier Vallon, Julian Whitelegge, John Whitmarsh, Bill Widger, X-ray diffraction, Jiusheng Yan, Huamin Zhang

\begin{abstract}
In this brief personal perspective, studies, mainly in my laboratory, directed toward understanding of the structure and function of the Fe-metalloprotein electron transport carriers of oxygenic photosynthesis are discussed. This level of understanding and the description of the events that led to this level are inevitably incomplete. The Fe-proteins considered are those, cytochromes $b-559$ (560), $f, b_{6}$, and $x$, and the Rieske [2Fe-2S] iron-sulfur protein, which have now been placed in a structural context as a result of X-ray diffraction analysis of crystals of Photosystem II and the cytochrome $b_{6} f$ complex and the Photosystem II reaction center.
\end{abstract}

Abbreviations: Cyt - cytochrome; DBMIB - 2,5 dibromo 3-methyl 6-isopropyl p-benzoquinone; DCMU - 3-(3,4dichlorophenyl)-1,1-(dimethylurea); $\mathrm{E}_{\mathrm{m}}$ - midpoint oxidation-reduction potential; EPR - electron paramagnetic resonance; FCCP - carbonyl cyanide- $p$-trifluoromethoxy-phenylhydrazone; FNR - ferredoxin:NADP ${ }^{+}$reductase; His - histidine; HP and LP - high and low potential; HPLC - high performance liquid chromatography; nm nanometer; PS - photosystem; SDS-PAGE; sodium dodecyl sulfate-polyacrylamide gel electrophoresis

\section{Introduction}

Over the period of our studies of the Fe-electron transport carriers in oxygenic photosynthesis, 'bread and butter' instrumentation in our laboratory has shifted from visible light difference spectrophotometry, initially involving the dual wavelength spectrophotometer (Figure 1, top) to include X-ray diffraction, carried out with highest accuracy at a synchrotron such as that at the advanced photon source at the Argonne National Laboratory (Figure 1, bottom). My mentor in photosynthesis studies, Warren Butler (Figure 2), had used the dual-wavelength spectrophotometer, developed by Britton Chance at the University of Pennsylvania Johnson Foundation (JF), to study light-induced redox changes of the chloroplast electron transport chain when he was on sabbatical at the JF in 1964. Warren was influenced by the ideas of Chance, and of Mordhay Avron (Avron and Chance 1965), who was also on sabbatical at the JF that year. (See a perspective by Chance, this issue.) Warren carried over the 'cross-over' concept to studies of chloroplast electron transfer when he moved to his new position as Professor in the Department of Biology at the new Revelle College at the University of California, San Diego (UCSD). Conceptually, the 'cross-over points' in the electron transport chain are the rate limiting electron transfer steps in the chain that are closely associated with 'phosphorylation sites', that is, sites coupled to ATP synthesis. According to present concepts, these crossover points would be intramembrane electron transfer steps linked to proton transfer within the membrane or across the membrane interface to the aqueous phase. 

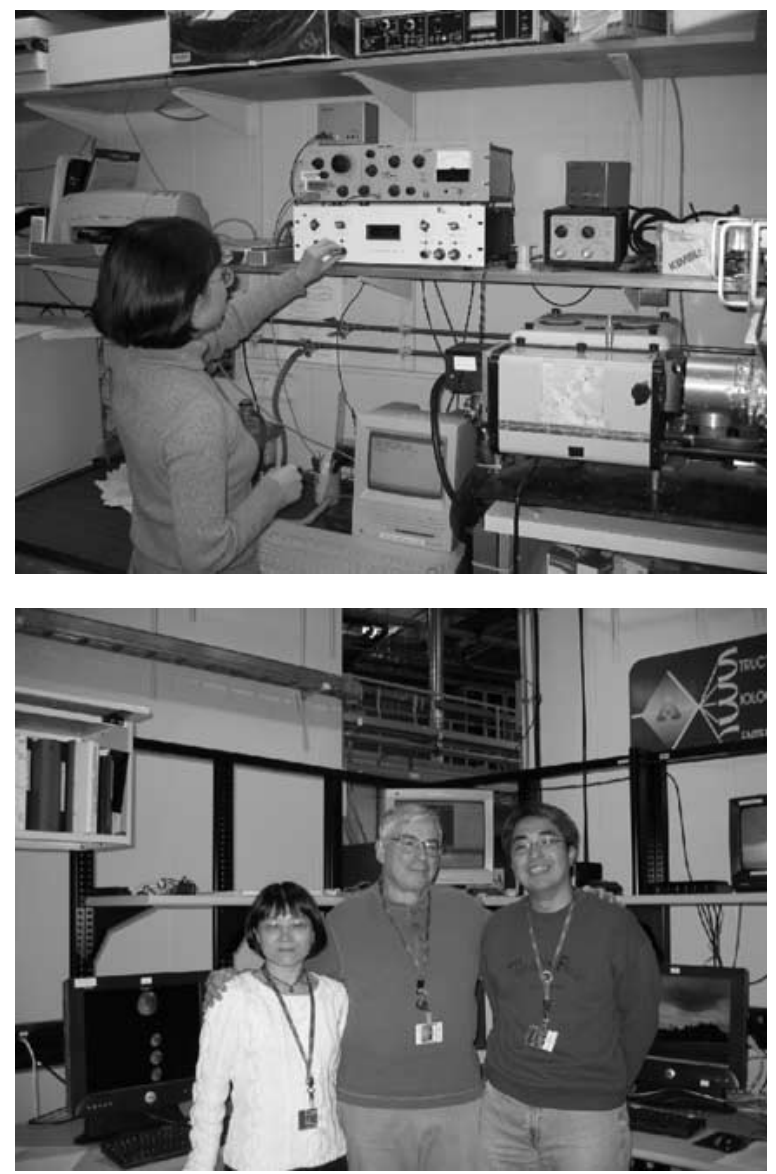

Figure 1. Top: The dual wavelength spectrometer is shown in a measurement of the electron transfer activity of isolated cytochrome $b_{6} f$ complex by Huamin Zhang. Huamin recently solved the problem of crystallization of the cytochrome $b_{6} f$ complex from the thermophilic cyanobacterium, M. laminosus (Zhang et al. 2003). The instrument was built by the author (WAC) in 1968 from individual components including a phase-sensitive amplifier following the design of Warren Butler, to measure cytochrome turnover. This somewhat ancient instrumentation is maintained and utilized partly because it still works well and partly to show any possible visitors from the Congress or the Government Office of Management and Budget that government funds are spent parsimoniously in academia for basic research. Bottom: Huamin Zhang and Genji Kurisu who, with Professor Janet L. Smith, carried out the crystallographic analysis of the cytochrome $b_{6} f$ complex from M. laminosus, with the author (WAC, in the center) at the SBC-19ID beam line of the synchrotron at the Advanced Photon Source, Argonne National Lab, Illinois, collecting X-ray diffraction data from crystals of the cyt $b_{6} f$ complex, with help from F. Rotella and N. Duke (Photos provided by Huamin Zhang).

Avron and Chance (1965) had shown that the steady-state reduction of cytochrome (Cyt) $f$ in chloroplasts under continuous actinic red light illumination behaved as though the cytochrome is on the oxidizing side of a cross-over point. The redox behavior

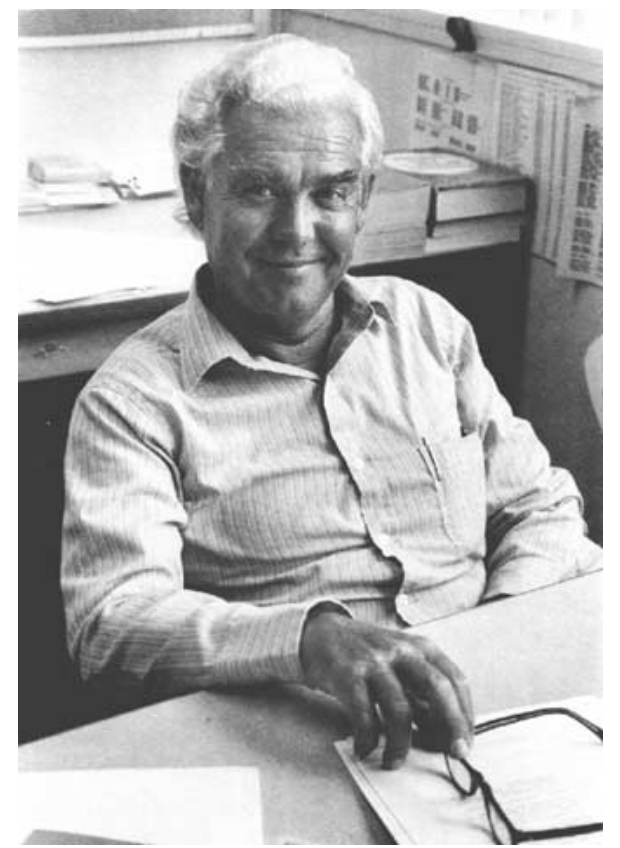

Figure 2. Warren L. Butler at his desk studying cryptic data on light-induced redox changes of the chloroplast $b$-cyto-chromes provided by the author (WAC) (photo provided by Govindjee).

of Cyt $f$ in the 'cross-over experiment' is a consequence of a rate-limiting step on the lumen-side membrane interface associated with the oxidation in the high-potential chain of plastoquinol, $\mathrm{PQH}_{2}$, to plastosemiquinone, $\mathrm{PQH}^{\bullet}$, and the linked proton transfer. A proton and electron are transferred to the Rieske iron-sulfur ([2Fe-2S]) protein, which is located at the lumen or $p$-side membrane interface (Kurisu et al. 2003; Stroebel et al. 2003). The millisecond half-time for reduction of Cyt $f$ is the slowest observable electron transfer step in the highpotential chain. The actual rate-limiting step, which is not readily observable spectroscopically, may be the electron transfer from plastoquinol to the $[2 \mathrm{Fe}-2 \mathrm{~S}]$ cluster (Crofts et al. 2000).

At the University of California at San Diego (UCSD) in late 1965, using dual wavelength difference spectrophotometry, Warren Butler wished to extend the studies in such cross-over experiments to the $b$-cytochrome that he believed, by analogy with the mitochondrial respiratory chain where the presence of such a cytochrome was known from spectrophotometric studies such as those of Chance and Williams (1956), to be present in chloroplast chain. It was somewhat of a surprise to find two spectral components, 
$b$-560 and $b$-563 (Cyt $b_{6}$ ), which were inferred to arise from the reduced $\alpha$-band peaks of two $b$-cytochromes (Cramer and Butler 1967). Neither spectral component showed the behavior that should occur if they were located on the reducing side of a cross-over site in a linear electron transfer chain. In retrospect, the cross-over behavior of the Cyt $b$ is mitochondria is surprising because it is located in the Cyt $b c_{1}$ complex whose Cyt $b$ core is conserved relative to Cyt $b_{6}$, as are the basic functions of the Cyt $b c_{1}$ and photosynthetic Cyt $b_{6} f$ complexes.

\section{Cytochrome $b$-559}

Jan Anderson and Keith Boardman saw a difference spectrum peak at $559 \mathrm{~nm}$ in a Photosystem II (PS II)-enriched fraction that they associated with a $b$ type cytochrome (Boardman and Anderson 1967). The association with PS II was prescient, as shown by the subsequent isolation of the PS II core by Nanba and Satoh (1987; see Satoh 2003). However, there is some question about the correctness of the '559 label,' which has been the subsequent designation of this cytochrome in the literature. The peak of the $\alpha$-band peak in purified Cyt $b-559$ (Widger et al. 1984a) was subsequently found by Gun-Sik Tae and Paul Furbacher to be very close to $560 \mathrm{~nm}$ in stromal membranes that were antigenically cross-reactive with antibody made to the PS II cytochrome traditionally designated as Cyt b-559 (Tae et al. 1993). Thus, we think that the proper spectroscopy-based nomenclature for the Cyt $b-559$ should actually be ' $b-560$.' This change in nomenclature is hereby proposed. To avoid confusion, the commonly used notation, Cyt $b$ 559 , will be followed until there is agreement on the nomenclature.

\section{Structure-function of cytochrome $b$-559}

Much is now known about the structure and structural environment of Cyt $b-559$. The function is still unclear. The critical structure-based findings on Cyt $b$-559 are:

(i) Cyt $b-559$ is localized in the core of the PS II reaction center, where Cyt $b-559$ was identified as a low molecular weight ca. $10 \mathrm{kDa}$ polypeptide (Nanba and Satoh 1987), which is the $\alpha$-subunit of the heterodimeric $(9 \mathrm{kDa}+4 \mathrm{kDa})$ protein (Widger et al. 1985). (ii) After the putative gene was located in the spinach plastid chromosome (Herrmann et al. 1984; Westhoff et al. 1985), determination of the Nterminal sequence of the $\alpha$-subunit of Cyt $b-559$ purified by Widger et al. (1984a) led to identification and the gene sequence of the Cyt $b$ 559, which was done when Bill Widger visited Reinhold Herrmann's lab (Herrmann et al. 1985). The DNA sequence coding for the Cyt $b-559$ showed two contiguous genes coding for two small polypeptides of 83 and 39 amino acids, the $\alpha-$ (psbE) and $\beta$-(psbF) subunits, each containing a putative single 20-25 residue hydrophobic putative trans-membrane helix containing only uncharged residues.

(iii) A bis-histidine heme ligation was inferred from the presence in each $\alpha$ - and $\beta$-polypeptide subunit of a single histidine residue that is five residues from an Arg residue at the $\mathrm{N}$-terminal end of the proposed trans-membrane helix membrane and the membrane polar interface (Widger et al. 1985), and through electron paramagnetic resonance (EPR) and resonance Raman spectral analyses carried out in Jerry Babcock's lab (Babcock et al. 1985). An experimental problem with studies on this cytochrome is that the smaller $4.3 \mathrm{kDa} \beta$-subunit is not usually visible on sodium dodecyl sulfate polyacrylamide gel electrophoresis (SDS-PAGE) of samples that included the cytochrome.

(iv) A $1: 1$ ratio of $\alpha: \beta$ subunits was determined from reversed phase high-performance liquid chromatography (HPLC) analysis of purified complex (Widger et al. 1985). The presence of only one histidine residue on each of the $\alpha$ - and $\beta$-subunits, which is in the same position in each polypeptide, close to the stromal-side polar interface, implied that the heme must be ligated by a two histidines contributed by each $\alpha$ - and $\beta$-polypeptide subunit, forming a $13 \mathrm{kDa}$ mono-heme $\alpha-\beta$ heterodimer, with the heme on the stromal side of the membrane. This structural model for orientation of the Cyt $b-559$ in the membrane was confirmed by Gun-Sik Tae working collaboratively with Olivier Vallon and Laurie Bogorad, using peptide-specific antibodies, by proteolysis of the $\alpha$ - (Tae et al. 1988; Tae and Cramer 1989; Vallon et al. 1989) and $\beta$-polypeptides in right-side out and inside-out thylakoid membranes (Tae and Cramer 1994).

(v) Early on, the midpoint redox potential $(+0.4 \mathrm{~V})$ of most of the cytochrome in 'fresh' chloroplasts 
was found to be anomalously positive. Much was made in our lab and others of a low potential form $\left(\mathrm{E}_{\mathrm{m}}=+50 \mathrm{mV}\right)$ of the cytochrome (Fan and Cramer 1970) that was found by Herbert Böhme and Ellen Fan in thylakoid membranes damaged by age, heat, protease (trypsin), or treatment with high concentrations of carbonyl cyanidep-trifluoromethoxy-phenylhydrazone (FCCP) and hydroxylamine (Cramer et al. 1971). The structural basis and functional significance of the low potential state is still not well understood. Jerry Babcock found a small difference in the $g$ values of high- and low-potential forms of the membrane-bound cytochrome $\left(\mathrm{g}_{\mathrm{z}}(\mathrm{HP})=3.08 ; \mathrm{g}_{\mathrm{z}}\right.$ $(\mathrm{LP})=2.94)$ that was interpreted in terms of a change in the orientation of the planes of the 2 ligating imidazole rings (Babcock et al. 1985). Another explanation, not necessarily structurally independent, is based on local dielectric properties of the membrane. Lev Krishtalik and Dmitri Cherepanov suggested that the very positive $E_{m}$ of the cyt $b-559$ could be attributed to the position of the heme in a stromal-side hydrophobic niche, where it is close to the N-terminus of the $\alpha$ - and $\beta$-polypeptides and thereby influenced by the positive dipole potential of the aligned $(\mathrm{N}-$ side stromal) parallel $\alpha$ - and $\beta$-polypeptide chains (Krishtalik et al. 1993). The high to low potential $-300 \mathrm{mV}$ transition of the cytochrome midpoint potential, from high to low potential, caused by heat or trypsin treatment was attributed to membrane damage and loss of the local hydrophobic niche (Krishtalik et al. 1993), and perhaps a contribution from changes in orientation of the orthogonal histidine ligands (Babcock et al. 1985).

(vi) The $\alpha-\beta$ heterodimeric structure and stromal side location of the heme of Cyt $b-559$ in the PS II reaction center has been confirmed in the crystal structure determination of the reaction center dimer purified from cyanobacteria to a resolution of 3.7-3.8 $\AA$ (Zouni et al. 2001; Kamiya and Shen 2003), although the orientation of the histidine side chains of the cytochrome cannot be determined at this resolution. The X-ray structure showing one stromal-side heme appears to have settled any question about the heterodimeric nature of Cyt $b$ 559. However, the functional significance of the high- and low-potential states of the cytochrome is still not known, and this function of the cytochrome continues to be largely a mystery. Later,
Peter Horton made the point that there may be a mid-potential cytochrome or a continuum of potentials.

(vii) A problem with understanding the function of the Cyt $b-559$, which still persists today, is summarized in a comment that was made by Bessel Kok about the slow (many seconds) light-induced redox changes of the Cyt $b-559$. Bessel commented to me at a Gordon Research Conference (GRC) in the mid 1970s that 'I couldn't show turnover of that cytochrome any faster than I could lick the spit off the end of my nose.' In those good old days at the GRC, a lot of truth was conveyed by Bessel, George Cheniae, and Warren in the wee hours, over a bottle of Jack Daniels or worse. The slow redox turnover of Cyt $b-559$ in which the cytochrome is oxidized by PS II might allow it to participate in processes of photoprotection (Stewart and Brudvig 1998; Barber 2002) which provided a role for the fundamental observation of photooxidation of the Cyt $b-559$ at liquid nitrogen temperature $(77 \mathrm{~K})$ by PS II (Knaff and Arnon 1969).

John Whitmarsh was able to provide a partial answer to Bessel by demonstrating light-induced reduction of the Cyt $b-559$ by PS II via the PQ pool with characteristic times $\left(\mathrm{t}_{1 / 2}=100 \mathrm{~ms} ; 25 \mathrm{~ms}\right.$ in the presence of 2,5 dibromo 3-methyl 6-isopropyl p-benzoquinone (DBMIB) and ferricyanide) that are appreciably faster than in the control experiment suggested by Bessel (Whitmarsh and Cramer 1978). The role of diffusible quinone in the redox interaction of $\mathrm{Q}_{\mathrm{B}}$ (the secondary plastoquinone acceptor of PS II) and Cyt $b-559$ was also demonstrated by Peter Horton and John, who showed that oxidation of the Cyt $b-559$ occurred in thylakoid membranes at a low $\mathrm{pH}$, where the quinone couple assumed a high potential. The oxidation was blocked by the quinol analogue inhibitor, 3-(3,4dichlorophenyl)-1,1-(dimethylurea) (DCMU) (Horton and Cramer 1975; Horton et al. 1976). If I had known the distances of separation between Cyt $b$-559 and its closest redox partners, approximately $28 \AA$ centercenter between $\mathrm{Q}_{\mathrm{B}}$ and the $b$-559 heme, and $27 \AA$ between the heme and chlorophyll Z (on D2 protein), which were shown in the crystal structure of PS II (Zouni et al. 2001), I would not have felt so badly at that time about Bessel's comment. The present structure data show that the redox reactions between the $\mathrm{Q}_{\mathrm{B}}$ acceptor of PS II and Cyt $b-559$ cannot be very fast. 


\section{Other suggestions for the function of cytochrome $b$-559}

It is probably Warren Butler who first used the term 'enigmatic' for the function of the Cyt $b$-559 (Butler 1978). Twenty-five years later, it remains so, although there are some promising hypotheses (Stewart and Brudvig 1998). The unique presence of Cyt $b$-559 in the $\mathrm{O}_{2}$-evolving PS II reaction center compared to the other reaction centers suggests that it has some role connected with the presence of $\mathrm{O}_{2}$ (Butler 1978; Shuvalov et al. 1995). This idea has been discussed in the context of EPR studies of the PS II D1/D2/ Cyt $b$ 559 complex (Shuvalov et al. 1995). The implication of the latter studies is that the Cyt $b-559$ might participate in reactions of water splitting. However, the stromal side location of the Cyt $b$-559 heme (Zouni et al. 2001) is on the wrong side of the membrane to participate in this pathway. One other function that could correlate with the presence of $\mathrm{O}_{2}$ evolution is a role of Cyt $b$-559 as an intra-membrane oxygen transporter. This hypothesis is based on the following: (i) The oxygen tension must be elevated in the membrane, and perhaps even higher near its source in PS II, because $\mathrm{O}_{2}$ is very soluble in hydrophobic environments (approximately 10 times more soluble in dioxane than in water); (ii) Double bonds of lipid fatty acid chains are labile toward $\mathrm{O}_{2}$-mediated peroxidation; (iii) The fatty acid chains of the thylakoid membrane are highly unsaturated; (iv) The heme is located on the periphery of PS II, facing toward the lipid; (v) The heme is oriented normal to the membrane plane, with one end close to the lumen-side aqueous interface, and the other close to the center of the membrane where the concentration of $\mathrm{O}_{2}$ would be high; (vi) It was argued that the EPR spectrum of a low-potential high-spin form of $b-559$ was similar to that of catalase with a hydroxyl ligand (Shuvalov et al. 1995).

\section{Mechanism of action of oxygen transporter}

It is hypothesized that Cyt $b-559$ can act as a transporter of intra-membrane $\mathrm{O}_{2}$ through oxygen-induced displacement of one of the heme histidine ligands by $\mathrm{O}_{2}$. The histidine displacement would occur as a result of a high concentration of $\mathrm{O}_{2}$ that is present in the membrane under high intensities of illumination. In this model, this 'histidine-flip' cycle would be completed by a religation of the heme by the wayward histidine, which would concomitantly displace an $\mathrm{O}_{2}$ molecule to the stromal-side aqueous phase.

The histidine displacement, the resulting coordination of the heme by $\mathrm{O}_{2}$, and perhaps the transient high spin signal would not have been detected in the usual visible (VIS) or EPR spectrometry experiment because these experiments usually involve high chlorophyll concentrations and resulting low effective intensities of actinic light. An experiment that would test this mechanism would be EPR and VIS spectrophotometric analysis of the Cyt $b-559$ spectra in the PS II reaction centers in the presence of hyperbaric $\mathrm{O}_{2}$.

\section{The cytochrome $b_{6} f$ complex}

Early data with structure implications for the Cyt $b$ core of Cyt $b c$ complexes in chloroplasts, mitochondria, and photosynthetic bacteria were derived from a pre-genomics era genomics analysis. During a visit to our lab by Achim Trebst in February 1983, Achim, Bill Widger and I analyzed the amino acid sequence of Cyt $b_{6}$ and subunit IV obtained by Reinhold Herrmann, and compared it with the several sequences existing at that time of Cyt $b$ from the Cyt $b c_{1}$ complex of mitochondria and photosynthetic bacteria. 'Eyeball hydropathy analysis' revealed that the length and distribution of 20-25 residue hydrophobic segments was the same in the Cyt $b$ from all these sources (Widger et al. 1984b). This analysis was shown to be quantitatively accurate by calculating an auto-correlation function, the same function that is used by astronomers to distinguish pulsars from random background flicker in the sky light, to the Kyte-Doolittle hydropathy functions (Kyte and Doolittle 1982). From the sequence and hydropathy analysis and comparisons, Bill Widger built a revealing model of the consecutive trans-membrane helices in Cyt $b\left(b c_{1}\right)$ and Cyt $b_{6}$ using all the available cardboard cores from the toilet paper in the nearby men's rooms. From the resulting 'high-resolution' model, it was clear that 2 of the trans-membrane helices contained 2 histidine residues, separated by 13-14 residues, one of the His in each helix on each side of the membrane.

The cardboard display made it clear that two bishistidine coordinated hemes cross-linked the same two helices, one His pair on each side of the membrane. With important input from Tony Crofts on the identity (the fourth, not the fifth) of the downstream second trans-membrane helix (Crofts et al. 1990), it became clear that the two hemes bridge the B and $\mathrm{D}$ (second and fourth) trans-membrane helices of the 
N-terminal four helices of the Cyt $b\left(b c_{1}\right)$ subunit. These N-terminal four helices contain the hemebinding domain, which corresponds to Cyt $b_{6}$ that has only four trans-membrane helices. It was pointed out that the motif of bis-histidine heme ligation arising from cross-linking of 2 trans-membrane helices is also present in the coordination of heme $a$ in subunit I of cytochrome oxidase, as initially predicted by the amino acid sequence of its subunit I, and subsequently described in its X-ray structure (Iwata et al. 1995; Tsukihara et al. 1995). It is likely to be a recurring structure motif of intramembrane heme proteins.

In a 1984 paper on this subject (Widger et al. 1984b), which I was proud to have sponsored by Warren Butler, we noted the conserved PEWY (proline-glutamic acid-tryptophan-tyrosine) sequence, in which the $\mathrm{E}$ has subsequently been implicated in the lumen-side pathway of proton transfer from ubiquinol in the bacterial Cyt $b c_{1}$ complex (Crofts et al. 1999). Given the apparent split of the 8 transmembrane helix mitochondrial Cyt $b$ to a 4 helix Cyt $b_{6}$ and a 3-helix subunit IV, the questions were: (i) why was the apparently highly conserved mitochondrial and photosynthetic bacterial Cyt $b$ gene divided in approximately half in oxygenic photosynthesis, where Cyt $b_{6}$ is the N-terminal half, the four helix heme-binding homologue of the mitochondrial cytochrome; (ii) was a relatively large 8 -helix protein split into 2 fragments, subunit IV and Cyt $b_{6}$ (Widger et al. 1984b), or were Cyt $b_{6}$ and subunit IV fused to form the 8-subunit Cyt $b$ of the Cyt $b c_{1}$ complex (Schütz et al. 1994)? Guenther Hauska and Nathan Nelson subsequently inferred that the nature of the $b$ cytochrome transition in evolution was a fusion of the Cyt $b_{6}$ and subunit-IV genes in the primitive oxygenic photosynthetic genome to yield the larger Cyt $b$ gene in the photosynthetic bacterium and the respiratory chain (Schütz et al. 1994). (For a historical account of the isolation of Cyt $b_{6} / f$ complex, see Hauska, this issue.)

\section{Pathway and mechanism of quinol oxidation}

Tony Crofts, Pierre and Anne Joliot have made major contributions to our understanding of the pathways of lumen-side plastoquinol oxidation in the Cyt $b_{6} f$ complex and formation of the proton electrochemical potential. The details of the bifurcated high and low potential pathways of plastoquinol oxidation in the Cyt $b_{6} f$ complex continue to unfold, as witnessed by the recent discovery in the low potential chain of a new heme, heme $c_{i}$ (Stroebel et al. 2003), or heme $x$ (Kurisu et al. 2003) in the crystal structures of the Cyt $b_{6} f$ complex (see below), which appears to be the terminal electron acceptor on the stromal side of the membrane. In the 'traditional' $Q$ cycle mechanism, the low potential pathway operates by transfer of an electron from the anionic semiquinone, formed by transfer of an electron to the Rieske [2Fe-2S] protein and two $\mathrm{H}^{+} \mathrm{s}$ to the $p$-side aqueous phase, and then one electron to heme $b_{p}$ and through to heme $b_{n}$ on the stromal side of the membrane. A new wrinkle in this pathway is implied by the proximity of heme $x$ to heme $b_{n}$. (See Tony Crofts, this issue, for a discussion of the 'modified Q-cycle'.)

\section{On the use of the notation $b_{p}$ and $b_{n}$}

The notation $b_{p}$ and $b_{n}$ for the two trans-membrane hemes is not commonly used. The other notations are: (a) $b_{i}$ and $b_{o}$ (i.e., $b$, respectively, inside and outside) and $b_{h}$ and $b_{1}$ (i.e., $b$, respectively high- and low-midpoint redox potential). The former notation is didactically unhelpful in a comparison of respiratory and photosynthetic electron transport chains, or the Cyt $b c_{1}$ and Cyt $b_{6} f$ complexes, as the 'in' side of the membrane in mitochondria is 'out' in chloroplasts, and vice versa; (b) The problem with the notation $b_{h}$ and $b_{1}$ is that the magnitude of the difference between the 'high' and 'low' potentials of the two hemes in thylakoid membranes is debated in the literature. There is agreement in the literature that the potentials of the two hemes are well separated in isolated complex. However, it is also well known that the complex is fragile. The question is whether the two potentials are sufficiently well resolved in situ in thylakoid membranes. On the one hand, they were well separated in the studies of Kramer and Crofts (1993). On the other hand: (a) our lab found in anaerobic redox titrations of thylakoid membranes that the $\Delta E_{m}$ between the two $E_{m}$ values was approximately $+50 \mathrm{mV}$ and could not be resolved (Girvin and Cramer 1984; Furbacher et al. 1989); (b) Rich et al. (1991) were unable by redox titrations of chloroplast membranes and quantitative statistical analyses of the data to assign different midpoint potentials to the two $b-563$ hemes. It is our opinion that it is best to have a notation that is readily understandable to mitochondriologists as well as photosynthetikers, and is not dependent on a small or debatable experimental difference. It is better to have the notation based on a 
difference, electrochemically positive $(p)$ and negative $(n)$, that is unambiguous and well known. Thus, we choose the notation, $b_{p}$ and $b_{n}$, for the two b-hemes on the two sides of the membrane in chloroplasts, mitochondria, and photosynthetic bacteria.

\section{The road to the crystal structure of the $b_{6} f$ complex}

The first preparation in our lab of purified Cyt $b_{6} f$ complex from spinach thylakoids by Mick Black and Bill Widger (Black et al. 1987) was characterized by us as 'highly active' and 'large-scale.' Another prominent feature of this preparation was the disproportionately high intensity of the Coomassie blue-stained band attributed to Cyt $f$ in the SDS-PAGE. Later, Huamin Zhang found that the unusually large width of this band on the SDS-PAGE could be attributed to the presence of ferredoxin-NADP ${ }^{+}$reductase (FNR). In retrospect, the 'high' activity of the complex purified at this time was $1 / 8-1 / 10$ that of the present preparation from thermophilic cyanobacterium, Mastigocladus laminosus (M. laminosus) that was ultimately used for successful crystallization in August, 2002. The subsequent evolution of our purification owes a lot to developments of methodolgy by G. Hauska and Richard Malkin. The goal of 'large-scale' turned out to be almost meaningless, as this hetero-oligomeric integral membrane protein complex, like most others, cannot be frozen and stored because they cannot be successfully crystallized after freeze/thaw. The present focus on high level expression of membrane proteins may similarly be receiving too much emphasis. The successful crystallization of the Cyt $b_{6} f$ complex from M. laminosus by Huamin Zhang utilized 10-15 mg of pure complex for crystallization obtained every second week. One reason for the long road to the crystal structure is that the complex appears to be inherently unstable, as inferred from the ability of moderate alkaline $\mathrm{pH}(10.5-11)$ to extract the very hydrophobic subunits of the complex from the membrane (Szczepaniak et al. 1991; Cramer et al. 1992). Exposure to extreme alkaline $\mathrm{pH}$ (approx. 13) is the textbook method for selective removal from membranes of extrinsically bound proteins. The presence of the subunits of Cyt $b_{6} f$ in the membrane or supernatant fractions was documented by Western blotting. These western experiments were carried out by Andrzej Szczepaniak according to a quantitative protocol that one rarely sees in the extensive literature employing this kind of experimentation. Andrzej ran a time course of each blot five times and then plotted the averages along with standard deviations. I must say that, although the antibody transfer techniques have innate experimental variabilities, I have never seen western data presented in this quantitative manner. The reasons for the ability of relatively mild alkaline conditions to cause extrusion of the hydrophobic Cyt $b$ and subunit-IV polypeptides of the Cyt $b_{6} f$ complex are not known. This result implies that electrostatic interactions are extraordinarily important in the stabilization of the complex. These unusual interactions have not yet been explained. However, the instability of the $b_{6} f$ complex manifested in the alkaline $\mathrm{pH}$ extraction experiment may explain why it was not possible to obtain well diffracting crystals of the complex for the longest time.

\section{Chipping away at the structure: (I) cytochrome $f$}

During this time, we were able to obtain structures of the p-side soluble domains of Cyt $f$ and the Rieske iron-sulfur protein, and thus to 'chip away' at the structure of the whole complex. We followed studies of John Gray, who had prepared a soluble Cyt $f$ from chloroplasts from Rape, from the plant group Cruciferae (Gray 1978). This preparation contrasted with the Cyt $f$ prep from spinach which tended to aggregate. The Mr value of the soluble Cyt $f$ was smaller by 2000-3000 mass units relative to that seen for Cyt $f$ in thylakoid membranes. Andrzej Szczepaniak hypothesized, and realized from C-terminal sequencing, that the soluble nature of the Cruciferae Cyt $f$ was a result of cleavage of a hydrophobic C-terminal segment that included the membrane-spanning $\alpha$-helix. Andrzej utilized his solid training in botany/plant physiology to choose turnip as an available member of the Cruciferae family in the West Lafayette super markets.

It turned out that the turnip Cyt $f$ was specifically and uniquely proteolyzed during the exposure to organic solvent utilized for chlorophyll extraction. This proteolysis turned out to occur at the lumen-side membrane interface of the trans-membrane helix. It clipped 33 residues at the $\mathrm{C}$-terminal end, resulting in a 252 residue soluble fragment. As John Gray had shown, this fragment had a normal spectrum and redox potential. To have an endogenous protease acting propitiously instead of disruptively and destructively in a protein purification is very unusual. We thank 
John Gray for his fundamental studies on this system. The protease effect also illustrates an important phenomenon associated with membrane proteins and their purification and crystallization. Because membrane protein insertion into, and assembly on or within, membranes must involve a complicated series of processing events at the membrane surface, solubilized membrane proteins are likely to be contaminated with proteases. In our opinion, this is a likely cause of the difficulties involved in their successful crystallization. We have still not solved the problem of the origin of the protease activity associated with the purified Cyt $b_{6} f$ complex, even though we have been able to crystallize it.

The soluble Cyt $f$ preparation could be crystallized by Sergio Martinez, a graduate student in the laboratory of Janet Smith, and Andrzej using acetone as precipitant (Martinez et al. 1992). The development of the crystallization strategy proceeded rapidly because Sergio did something that all crystallographers should do when trying to solve the problem of protein crystallization. They should work through the purification procedure by themselves. Sergio did this and realized that, because acetone was used to precipitate Cyt $f$ in the purification, without any deleterious effects, it could be used as a precipitant in the crystallization. It was, and the structure could be solved from turnip to a resolution of $1.9 \AA$ (Martinez et al. 1994). The structure showed a unique $c$-type cytochrome and, in contrast to the b-cytochrome, completely different except for the heme binding signature, from the functionally analogous Cyt $c_{1}$ in the Cyt $b c_{1}$ complex. From structures of the soluble Cyt $f$ from the cyanobacterium, Phormidium laminosus provided by Derek Bendall and Beatrix Schlarb (Carrell et al. 1999) and the green alga Chlamydomonas (Sainz et al. 2000), this unique structure has been shown to be conserved in the evolution of oxygenic photosynthesis. One consequence of the difference between Cyt $f$ and Cyt $c_{1}$ is a different trajectory of smaller amplitude followed by the Rieske protein between its membrane proximal position and that proximal to the high potential Cyt $f$ or Cyt $c_{1}$ that is necessary for electron transfer (Yan and Cramer 2003).

The high resolution crystal structure of Cyt $f$ allowed 'intelligent mutagenesis' to be carried out in the transformable heterotrophic green alga, Chlamydomonas reinhardtii. Through mutagenesis, Glenda Soriano and Misha Ponamarev addressed the problem of the role of the 'basic positively charged patch' in Cyt $f$ in the electron transfer reaction between
Cyt $f$ and plastocyanin (Soriano et al. 1996, 1998). In stopped-flow measurements of the Cyt $f$-plastocyanin (PC) intermolecular electron transfer rate, Glenda and Misha could show that the basic residues, at the interface between the large and small domains, greatly facilitated the electron transfer, as predicted from calculations of the role of electrostatics in their docking of these electron transfer partners (Pearson et al. 1996; Soriano et al. 1997). However, when the rate of Cyt $f$ oxidation was measured in wild type cells of $C$. reinhardtii, and mutants in which the basic patch on Cyt $f$ was eliminated, there was no difference in the rate of Cyt $f$ oxidation. Thus, in vivo, in the crowded luminal space, long-range electrostatic attraction between Cyt $f$ and PC is not a limiting step. The docking site for effective electron transport is not specific. This is the only protein electron transfer system, of the many that have been studied in vitro, which has been analyzed in vivo and in vitro. Misha also used the Chlamydomonas system to solve the classical problem of the apparently small but anomalous difference in the spectra of Cyt $f$ in plants and green algae relative to cyanobacteria, where the reduced alpha band spectrum is red-shifted by $2 \mathrm{~nm}$ (Ponamarev et al. 2000). The difference rests in the identity of the residue in position 4, whether it is $\operatorname{Trp}$ (cyanobacteria) or Tyr/Phe (plants, green algae).

\section{Chipping away at the structure: (II) the Rieske iron-sulfur protein}

A soluble fragment, 139 residues of the 179 residue lumen-side Rieske iron-sulfur protein from spinach Cyt $b_{6} f$ complex was prepared by Huamin Zhang in 1996. Following a protocol similar to that of Thomas Link, used for purification of an analogous soluble fragment from the Cyt $b c_{1}$ complex (Link et al. 1992), Huamin used an exogenous protease, thermolysin, to cleave an $\mathrm{N}$-terminal 40-residue segment from the Rieske protein in the complex. Although there are differences in the $p$-side surface of the mitochondrial Cyt $b c_{1}$ complex, and the Cyt $b_{6} f$ complex from spinach, this strategy of cutting off at its roots the peripheral domain of an embedded membrane protein worked for both complexes. Huamin was able to obtain one good crystal of the truncated Rieske protein. This protein was sent to Janet Smith who was on sabbatical at the Grenoble synchrotron facility in France. The quality of the multiple anomalous dispersion obtained from the $2 \mathrm{Fe}$ atoms by Janet in this one crystal allowed Chris 
Carrell to obtain a high resolution $(1.8 \AA)$ structure of the soluble Rieske domain (Carrell et al. 1997). This meant that, in pieces, we now knew the structure of about $40 \%$ of the mass of the Cyt $b_{6} f$ complex.

During this period, the complex was better characterized biochemically and we began to learn the basics of membrane protein crystallization crystallography. I learned an enormous amount from a sabbatical in the laboratory of Hartmut Michel at the Max Planck Institute in Frankfurt, Germany.

Deru Huang and Mike Everly found that the active Cyt $b_{6} f$ complex was a dimer, and that, oddly (still oddly), it contains one molecule of bound chlorophyll $a$ (Huang et al. 1994). It was realized that the presence of a chlorophyll in the complex must require a proximal carotenoid. Huamin subsequently found a $\beta$ carotene present at an approximately $1: 1$ ratio with the chlorophyll (Zhang et al. 1999), which should have solved the problem of triplet state quenching. Huamin also wondered about the very broad, intense SDS-PAGE band associated with Cyt $f$, which was prominent in the first serious Cyt $b_{6} f$ prep that we did, from spinach (Black et al. 1987). Huamin found that the additional intensity in the SDS-PAGE arose from FNR bound to the Cyt $b_{6} f$ complex, which led to the suggestion of a complex, perhaps just transient, between the Cyt $b_{6} f$ complex and PS I that functions to reduce $\mathrm{NADP}^{+}$(Zhang et al. 2001).

The recently obtained crystal structure of the Cyt $b_{6} f$ complex from the thermophilic cyanobacterium, M. laminosus (Kurisu et al. 2003) was the result of many important biochemical contributions from many others, including D.S. Bendall, G. Hauska, T. Kallas, R. Malkin, J.-L. Popot, and C. de Vitry. (See Derek Bendall for a history of Cyt $f$, and Günter Hauska for a history of Cyt $b_{6} / f$ complex.) In our lab, the pondering of these contributions and the development of our methodology took approximately 100,000 person-hours of work. A photograph taken during this period shows $\mathrm{H}$. Michel pointing out to the author an error (one of many) of his crystallographic ways (Figure 3). Deru Huang had made a lot of progress on the preparation. We have not yet been able to crystallize the complex from spinach. Nor yet to obtain intact active complex from any transformable cyanobacterium. We did not want to work on the $C$. reinhardtii system because we knew that the groups of Jean-Luc Popot and Daniel Picot in Paris were using it. Deru was able to prepare intact active complex from the filamentous thermophilic cyanobacterium, M. laminosus, but could not obtain

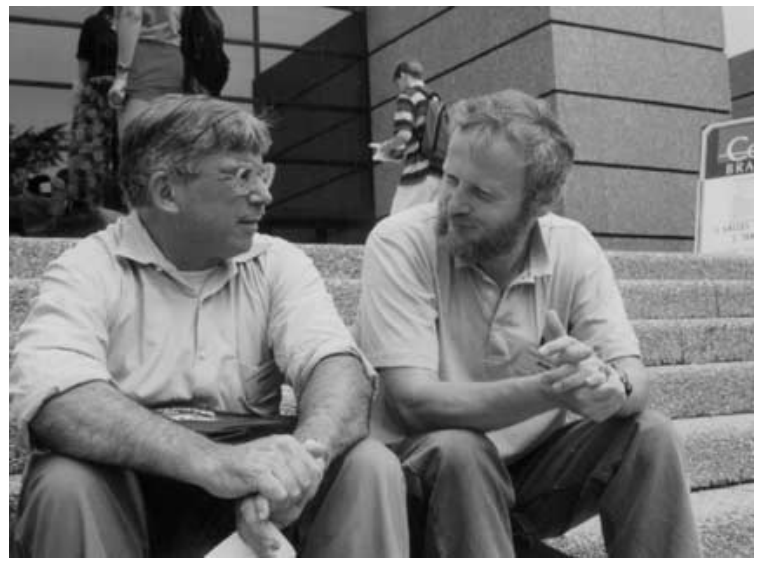

Figure 3. At Montpellier, France, Hartmut Michel is seen explaining to the author (WAC) a major flaw in the strategy of the latter for crystallization of integral membrane proteins (photo by Govindjee).

crystals that diffracted well. There were three subsequent major steps of progress: (1) Huamin realized that the crystals that we could obtain, only after 2-3 months of incubation, contained proteolyzed subunits, most obviously the Rieske protein; Huamin then started to systematically assay the stability of the complex incubated at room temperature; (2) We feared that the inability to crystallize was perhaps a consequence of the loss of one of the small Pet polypeptides that we did not routinely assay in SDS-PAGE. Based on the known cyanobacterial genome sequences, we thought that the complex might have lost a subunit. To answer this question, Julian Whitelegge at University of California at Los Angeles (UCLA) carried out a mass spectroscopic analysis of the complex (Whitelegge et al. 2002). This was a unique and original analysis of a hydrophobic membrane protein complex. The mass measurement involved the largest number of subunits and the largest range of masses yet determined. Not only did he find all of the 7 polypeptide components that we thought we had, but an additional small (3-4kDa) hydrophobic polypeptide, for a total of eight. Thus, we were reinvigorated, and as well by the arrival in the lab of Genji Kurisu. Genji had obtained a crystal structure an FNR-ferredoxin complex and wanted to crystallize and solve the complex of Cyt $b_{6} f$ and FNR from spinach; (3) In the summer of 2002, we realized that the complex was delipidated, and inferred that this was a cause of the destabilization of the complex. Thus, one could say that we had succeeded so well in our efforts to purify the complex that we had gone too far. Huamin, Jiusheng Yan, Genji, and I convened the 999th discussion of 
problems with the crystrallization of the complex. We decided that we should add lipid back to the complex. We could not decide on the lipid. We had a variety of synthetic lipids in our inventory for liposome and planar bilayer studies. We said jokingly to Huamin to pick the first lipid that you see in the freezer. She picked dioleolyl-phosphatidyl-choline and then made an inspired guess about the amount, 10 mol per mol Cyt $f$, that was added to the complex. Crystallization droplets were set up. Large $(0.2-0.4 \mathrm{~mm})$ orthogonal red-brown crystals appeared on the next day. This technique of 'lipid augmentation' in which the protein complex is 'over-purified' so that it is delipidated and the lipid content then restored by addition of synthetic lipid (Zhang et al. 2003) may have general applicability to the crystallization of other hetero-oligomeric integral membrane proteins.

The new structure of the complex from M. laminosus, in which a plastoquinone is seen on the $n$-side of the quinone exchange cavity, raises the question of the extent to which electron transfer through the low potential chain from the $p$ - to the $n$-side of the Cyt $b_{6} f$ complex is mediated by diffusion of plasto-semiquinone instead of the two trans-membrane $b$-cytochromes. It was implied by experiments of Mark Girvin in which he found that turnover of the Cyt $b_{6}$ was not required for generation of the slow light-induced electrochromic phase, which was not diminished by prior reduction of the $b$ cytochromes (Girvin and Cramer 1984; Girvin 1985). The existence of the semiquinone $p>n$ pathway has also been subsequently invoked to explain studies by Jiusheng Yan on light-induced redox changes of the Cyt $b_{6} f$ complex in an in vivo system, in which the amplitude of light-induced reduction of hemes $b$ was vanishingly small compared to that of Cyt $f$ oxidation in logarithmic phase cells of the cyanobacterium, Synechococcus 7002. Thus, the trans-membrane 'semiquinone cycle' (Joliot and Joliot 1994) may be an alternative pathway under in vivo conditions when the ambient intra-cellular oxidationreduction potential is very reducing. Regarding the semiquinone cycle, however, it must be said that it is peculiar to have the two trans-membrane $b$-hemes, $b_{\mathrm{p}}$ and $b_{\mathrm{n}}$ just dangling there doing nothing when the semiquinone cycle is operating.

After the first test diffraction experiments on lipidaugmented $M$. laminosus Cyt $b_{6} f$ complex turned out well, Genji dropped the project on crystallization of the spinach FNR-Cyt $b_{6} f$ complex to be able to work on the crystallographic analysis and structure solution
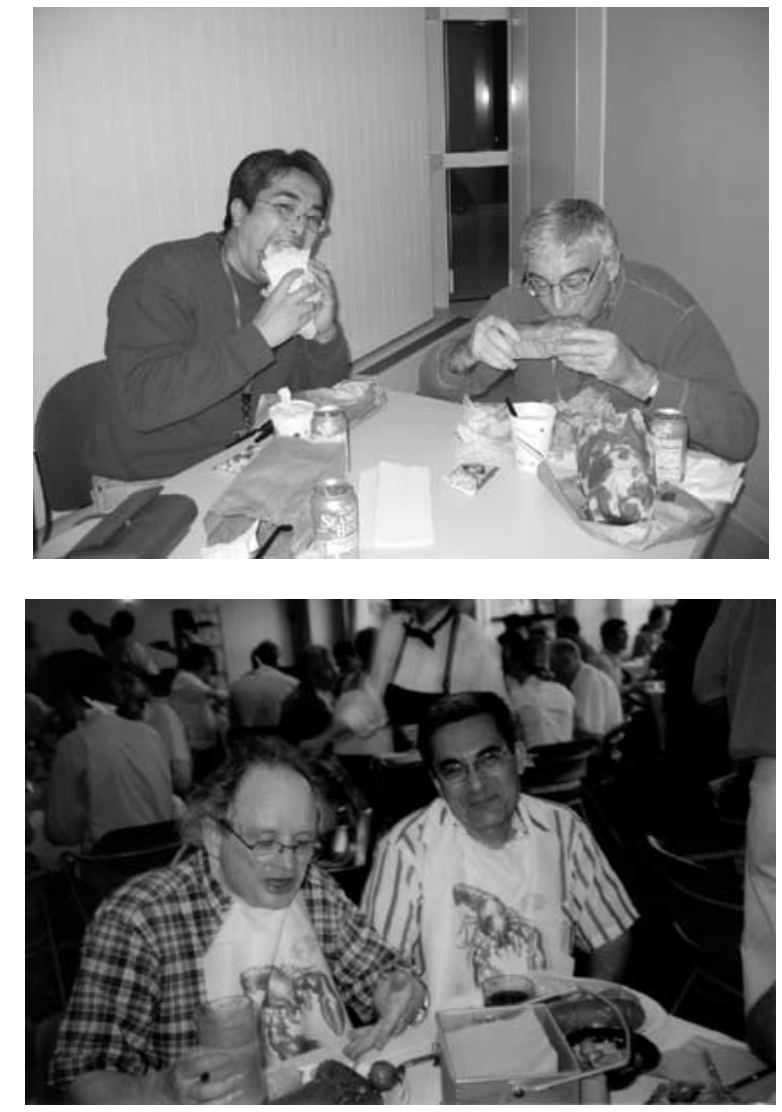

Figure 4. Chewing over the problems of the crystal structures of the $b_{6} f$ complex: (Top) G. Kurisu and W.A. Cramer and (Bottom) J.-L. Popot and F. Daldal, respectively, considering the complex from $M$. laminosus and $C$. reinhardtii while enjoying the APS pork sandwich and the GRC lobster. Top and bottom photos are by Huamin Zhang and Govindjee respectively. A photograph of the Mastigocladus and Chlamydomonas groups is shown in Figure 14 in Govindjee and Krogmann (this issue).

of the crystallized complex. With critical input from Professor Janet Smith, a structure with $3.0 \AA$ resolution was obtained (Kurisu et al. 2003). As always with such data, many questions are answered and new ones raised. It is probably too early to comment on the new questions raised by this structure. One general conclusion is that the similarity in the $C$. reinhardtii (Stroebel et al. 2003) and M. laminosus structures, derived from organisms perhaps a billion years apart on the evolutionary time scale is mind-boggling. One of the members, J.-L. Popot, of the C. reinhardtii Cyt $b_{6} f$ group, and two of the M. laminosus group, G. Kurisu and W. Cramer, are seen chewing over these problems (Figure 4).

One difference may be that the distance and conformational change of the Rieske $[2 \mathrm{Fe}-2 \mathrm{~S}]$ pro- 


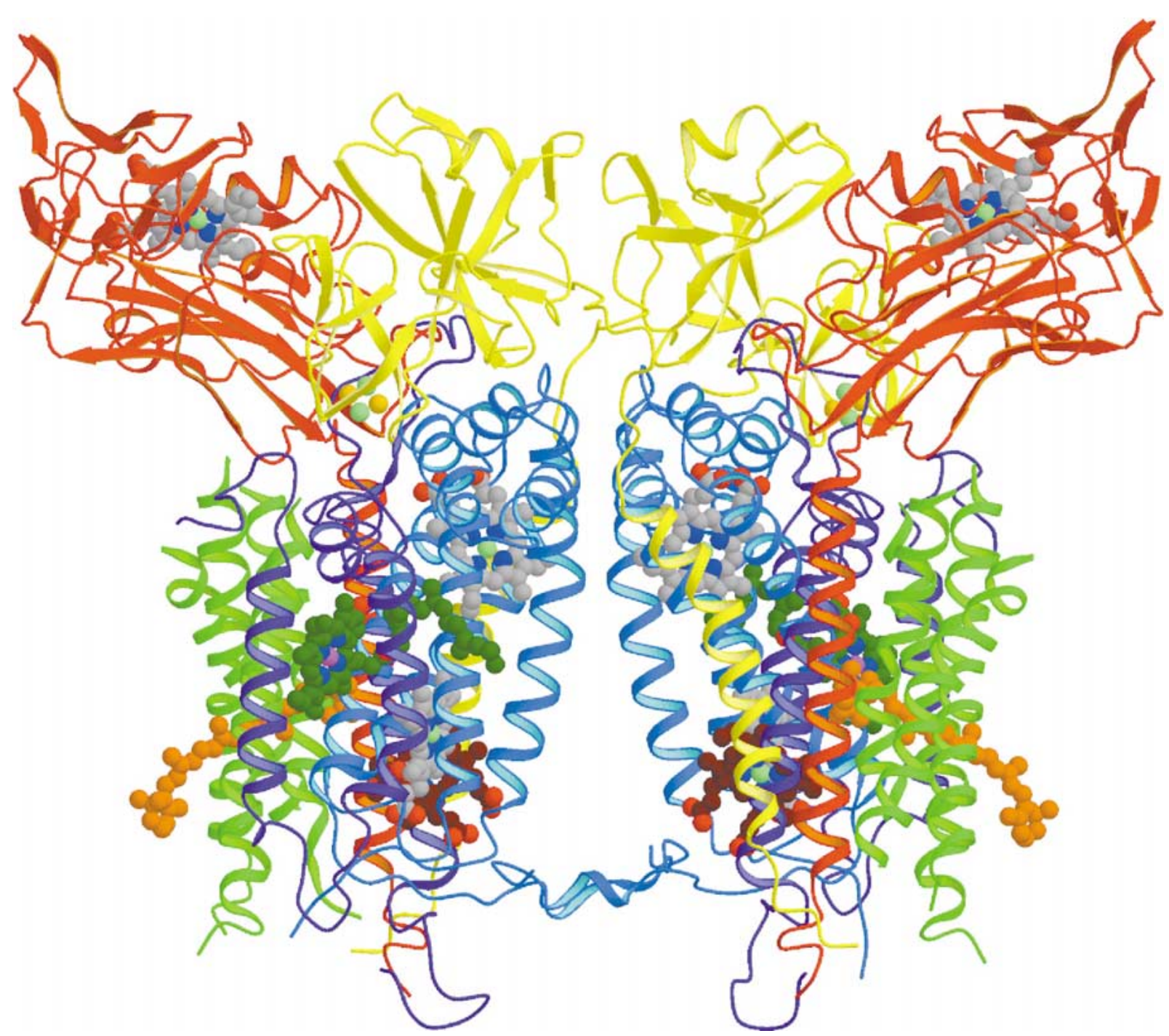

Figure 5. The curtain made of the 26 trans-membrane a-helices of the dimeric cytochrome $b_{6} f$ complex (Kurisu et al. 2003) has descended on the first part of the play. Eight natural prosthetic groups, 4 hemes colored gray (1 Cyt $f, 2 \mathrm{Cyt} b, 1$ heme $x, 1$ [Fe2S], 1 chlorophyll $a$ (green), $1 \beta$-carotene (orange), 1 bound plastoquinone) are seen in each half (monomer) of the dimer. The Cyt $f$ heme is readily accessible to Cyt $c_{6}$. The curtain is also seen to have a cavity, the 'quinone exchange cavity' between the two monomers of the dimeric structure (bottom-center). This cavity provides entry into the Cyt $b_{6} f$ complex of the lipophilic quinones that reside in the hydrophobic core of the membrane bilayer (M. Girvin et al. 1985; Ulrich et al. 1985). (Reproduced with permission of the American Association of the Advancement of Science.)

tein required for electron transfer from the $p$-side plastoquinol to the Cyt $f$ heme seems to be much smaller in M. laminosus than in the $C$. reinhardtii complex or the Cyt $b c_{1}$ complex, consistent with studies of Jiusheng Yan (Yan and Cramer 2003) in which he examined the consequences for activity of the complex of mutagenizing the hinge region of the Rieske protein.

The curtain, made of the 26 trans-membrane $\alpha$ helices in the $b_{6} f$ dimer, then descends on the first few Acts of this play (Figure 5).

\section{Acknowledgments}

I thank all of my close colleagues, Herbert Bohme, Achim Trebst, Peter Horton, John Whitmarsh, Bill Widger, Mark Girvin, Gun-Sik Tae, Mick Black, Andzrej Szczepaniak, Paul Furbacher, Deru Huang, Mike Everly, Lev Krishtalik, Misha Ponamarev, Glenda Soriano, Huamin Zhang, Jiusheng Yan, Genji Kurisu, and Janet Smith, who had a key role in working out some of the ironies of photosynthetic electron transport. We thank Professor Alison Butler for sup- 
plying pictures of Professor Warren L. Butler that were an inspiration during the writing of this article. The research described here has been supported over the years by the NSF (National Science Foundation), USDA (United States Department of Agriculture), or the NIH (National Institutes of Health) GM-38323. The latter was the source of support for studies that led to the crystal structure of the cytochrome $b_{6} f$ complex. Completion of this article was made possible by the persistent attention of Govindjee. The paper was edited by Govindjee and J. Thomas Beatty.

\section{References}

Avron M and Chance B (1965) The relation of light-induced oxidation-reduction changes in cytochrome $f$ of isolated chloroplasts to photophosphorylation. In: Thomas JB and Goedheer JC (ed) Currents in Photosynthesis, pp 455-463. Donker, Rotterdam, The Netherlands

Babcock GT, Widger WR, Cramer WA, Oertling W and Metz JG (1985) Axial ligands of cytochrome $b$-559: identification and requirement for a heme-cross-linked polypeptide structure. Biochemistry 24: 3638-3645

Barber J (2002) Photosystem II: a multisubunit membrane protein that oxidises water. Curr Opin Struct Biol 12: 523-530

Black MT, Widger WR and Cramer WA (1987) Large-scale purification of active cytochrome $b_{6} f$ complex. Arch Biochem Biophys 252: 655-661

Boardman NK and Anderson JM (1967) Fractionation of the photochemical systems of photosynthesis II. Cytochrome and carotenoid contents of particles isolated from spinach chloroplasts. Biochim Biophys Acta 143: 187-203

Butler WL (1978) On the role of cytochrome $b$-559 in oxygen evolution in photosynthesis. FEBS Lett 95: 19-25

Carrell CJ, Zhang H, Cramer WA and Smith JL (1997) Biological identity and diversity in photosynthesis and respiration: structure of the lumen-side domain of the chloroplast Rieske protein. Structure 5: 1613-1625

Carrell CJ, Schlarb BG, Howe CJ, Bendall DS, Cramer WA and Smith JL (1999) Structure of the cytochrome $f$ soluble domain from Phormidium laminosus: convergent evolution of the membrane-bound $c$-type cytochromes from respiratory and photosynthetic $b c$ complexes. Biochemistry 38: 9590-9599

Chance B (2004) The stopped-flow method and chemical intermediates in enzyme reactions - a personal essay. Photosynth Res 80: 387-400 (this issue)

Chance B and Williams GR (1956) The respiratory chain and oxidative phosphorylation. Adv Enzymol 17: 65-135

Cramer WA and Butler WL (1967) Light-induced absorbance changes of two $b$ cytochromes in spinach chloroplasts. Biochim Biophys Acta 143: 332-339

Cramer WA, Fan HN and Böhme H (1971) High and low potential states of the chloroplast cytochrome $b-559$ and thermodynamic control of non-cyclic electron transport. J Bioenerg 2: 289-303

Cramer WA, Engelman DM, von Heijne G and Rees DC (1992) Forces involved in the assembly and stabilization of membrane proteins. FASEB J 6: 3397-3402

Crofts AR, Yun CH, Gennis RB and Mahalingham S (1990) Prediction of structure for cytochrome $b$ from sequence data: what information is available from sequence comparison. In: Baltscheffsky $M$ (ed) Current Research in Photosynthesis, Vol III, pp 263-266. Kluwer Academic Publishers, Dordrecht/Boston/London

Crofts AR, Hong SJ, Ugulava N, Barquera B, Gennis RB, GuergovaKuras M and Berry E (1999) Pathways for proton release during ubihydroquinone oxidation by the $\mathrm{bc}_{1}$ complex. Proc Natl Acad Sci USA 96: 10021-10026

Crofts AR, Guergova-Kuras M, Kuras R, Ugulava N, Li J and Hong S (2000) Proton-coupled electron transfer at the $\mathrm{Q}_{0}$-site: what type of mechanism can account for the high activation barrier? Biochim Biophys Acta 1459: 456-466

Fan HN and Cramer WA (1970) The redox potential of cytochrome $b-559$ and $b-563$ in spinach chloroplasts. Biochim Biophys Acta 216: 200-207

Furbacher PN, Girvin ME and Cramer WA (1989) On the question of interheme electron transfer in the chloroplast cytochrome $b_{6}$ in situ. Biochemistry 28: 8990-8998

Girvin ME (1985) Electron and Proton Transfer in the QuinoneCytochrome $b$ - $f$ Region of Chloroplasts. Purdue University, West Lafayette, Indiana

Girvin ME and Cramer WA (1984) A redox study of the electron transport pathway responsible for generation of the slow electrochromic phase in chloroplasts. Biochim Biophys Acta 767: 29-38

Govindjee and Krogmann D (2004) Discoveries in oxygenic photosynthesis (1727-2003): a perspective. Photosynth Res 80: 15-57 (this issue)

Gray J (1978) Purification and properties of a monomeric cytochrome $f$ from Charlock. Eur J Biochem 82: 113-141

Herrmann RG, Alt J, Schiller B, Widger WR and Cramer WA (1984) Nucleotide sequence of the gene for apocytochrome $b-559$ on the spinach plastid chromosome: implications for the structure of the membrane protein. FEBS Lett 176: 239-244

Horton $\mathrm{P}$ and Cramer WA (1975) Acid-base induced redox changes of the chloroplast cytochrome $b$-559. FEBS Lett 56: 244-247

Horton P, Whitmarsh J and Cramer WA (1976) On the specific site of action of 3-(3,4-dichlorophenyl)-1,1-dimethylurea in chloroplasts: inhibition of a dark acid-induced decrease in midpoint potential of cytochrome $b-559$. Arch Biochem Biophys 176: 519-524

Huang D, Everly RM, Cheng RH, Heymann JB, Schägger H, Sled V, Ohnishi T, Baker TS and Cramer WA (1994) Characterization of the chloroplast cytochrome $b_{6} f$ complex as a structural and functional dimer. Biochemistry 33: 4401-4409

Iwata S, Ostermeier C, Ludwig B and Michel M (1995) Structure at $2.5 \AA$ resolution of the cytochrome $c$ oxidase from Paracoccus denitirificans. Nature 376: 660-669

Joliot P and Joliot A (1994) Mechanism of electron transfer in the cytochrome $b / f$ cycle of algae: the semiquinone cycle. Proc Natl Acad Sci USA 91: 1034-1038

Kamiya N and Shen JR (2003) Crystal structure of oxygen-evolving photosystem II from Thermosynechococcus vulcanus at $3.7 \AA$ resolution. Proc Natl Acad Sci USA 100: 98-103

Knaff DB and Arnon DI (1969) Light-induced oxidation of a $b$-cytochrome at $-189^{\circ} \mathrm{C}$ Proc Natl Acad Sci USA 63: 956-962

Kramer DM and Crofts AR (1993) Re-examination of the properties and function of the $b$ cytochromes of the thylakoid cytochrome bf complex. Biochim Biophys Acta 1184: 193-201

Krishtalik LI, Tae GS, Cherepanov DA and Cramer WA (1993) The redox properties of cytochromes $b$ imposed by the membrane electrostatic environment. Biophys J 65: 184-195 
Kurisu G, Zhang H, Smith JL and Cramer WA (2003) Structure and mechanism of the cytochrome $b_{6} f$ complex of oxygenic photosynthesis: tuning the cavity. Science 100: 6713-6723

Kyte J and Doolittle RF (1982) A simple method for displaying the hydropathic character of a protein. J Mol Biol 157: 105-132

Link TA, Hagen WR, Pierik AJ, Assmann AJ and von Jagow G (1992) Determination of the redox properties of the Rieske $[2 \mathrm{Fe}-2 \mathrm{~S}]$ cluster of bovine heart bc1 complex by direct electrochemistry of a water-soluble fragment. Eur J Biochem 208: 685-691

Martinez SE, Smith JL, Huang D, Szczepaniak A and Cramer WA (1992) Crystallographic studies of the lumen-side domain of turnip cytochrome $f$. In: Murata N (ed) Research in Photosynthesis, Vol. II, pp 495-498. Kluwer, Dordrecht

Martinez SE, Huang D, Szczepaniak A, Cramer WA and Smith JL (1994) Crystal structure of chloroplast cytochrome $f$ reveals a novel cytochrome fold and unexpected heme ligation. Structure 2: $95-105$

Nanba O and Satoh K (1987) Isolation of a photosystem II reaction center consisting of D1 and D2 polypeptides and cytochrome $b$-559. Proc Natl Acad Sci USA 84: 109-112

Pearson DC, Gross EL and David ES (1996) Electrostatic properties of cytochrome $f$ : implications for docking with plastocyanin. Biophys J 71: 64-76

Ponamarev MV, Schlarb B, Carrell CJ, Howe CJ, Smith JL, Bendall DS and Cramer WA (2000) Tryptophan-heme $\pi$-electrostatic interactions in cytochrome $f$ of oxygenic photosynthesis. Biochemistry 39: 5971-5976

Rich PR, Madgwick SA and Moss DA (1991) The interaction of duroquinol, DBMIB, and NQNO with the cytochrome $b_{6} f$ complex. Biochim Biophys Acta 1058: 312-328

Satoh K (2003) The identification of the photosystem II reaction center: a personal story. Photosynth Res 76: 233-240

Schütz M, Zirngibl S, le Coutre J, Büttner M, Xie DL, Nelson N, Deutzmann N and Hauska G (1994) A transcription unit for the Rieske FeS-protein and cytochrome $b$ in Chlorobium limicola combines characteristics of the bc1/ $b_{6} f$-types. Photosynth Res 39: 163-174

Shuvalov VA, Fiege R, Schreiber U, Lendzian F and Lubitz W (1995) EPR study of cytochrome in the D1D2 Cyt $b$-559 complex. Biochim Biophys Acta 1228: 175-180

Soriano G, Ponamarev M, Tae GS and Cramer WA (1996) Effect of the interdomain basic region of cytochrome $f$ on its redox reactions in vivo. Biochemistry 35: 14590-14598

Soriano GM, Krishtalik LI and Cramer WA (1997) Electrostatic effects on electron transfer kinetics in the cytochrome $f$-plastocyanin complex. Biophys J 73: 3265-3276

Soriano GM, Ponamarev MV, Piskorowsk R and Cramer WA (1998) Identification of the basic residues of cytochrome $f$ responsible for the electrostatic docking interaction in vitro: relevance to the electron transfer reaction in vivo. Biochemistry 37: 15120-15128

Stewart DH and Brudvig GW (1998) Cytochrome $b_{559}$ of photosystem II. Biochim Biophys Acta 1367: 63-87

Stroebel D, Choquet Y, Popot JL and Picot D (2003) An atypical heme in the cytochrome $b_{6} f$ complex. Nature 426: 413-418

Szczepaniak A, Huang D, Keenan TW and Cramer WA (1991) Electrostatic destabilization of the cytochrome $b_{6} f$ complex in the thylakoid membrane. EMBO J 10: 2757-2764

Tae GS and Cramer WA (1989) Lumen-side topography of the $\alpha$ subunit of the chloroplast cytochrome $b-559$. FEBS Lett 259: $161-164$
Tae GS and Cramer WA (1994) Topography of the heme prosthetic group of cytochrome $b$-559 in the photosystem II reaction center. Biochemistry 33: 10060-10068

Tae GS, Black MT, Cramer WA, Vallon O and Bogorad L (1988) Thylakoid membrane protein topography: transmembrane orientation of the chloroplast cytochrome $b-559 \mathrm{ps} b \mathrm{E}$ gene product. Biochemistry 27: 9075-9080

Tae GS, Everly RM, Cramer WA, Madgwick SA and Rich PR (1993) On the question of the identity of cytochrome $b-560$ in thylakoid stromal membranes. Photosynth Res 36: 141-146

Tsukihara T, Aoyama H, Yamashita E, Tomizaki T, Yamaguchi H, Shinzawa-Itoh K, Nakashima R, Yaono R and Yoshikawa $\mathrm{S}$ (1995) Structures of metal sites of oxidized bovine heart cytochrome oxidase. Science 269: 1069-1074

Ulrich EL, Girvin ME, Cramer WA and Markley JL (1985) Location and mobility of ubiquinones of different chain lengths in artificial membrane vesicles. Biochemistry 24: 2501-2508

Vallon O, Tae Cramer WA, Simpson D, Hoyer-Hansen G and Bogorad L (1989) Visualization of antibody-binding to the photosynthetic membrane: the transmembrane orientation of cytochrome $b$-559. Biochim Biophys Acta 975: 132-141

Westhoff P, Alt J, Widger WR, Cramer WA and Herrmann RG (1985) Localization of the gene for apocytochrome $b-559$ on the plastid chromosome of spinach. Plant Mol Biol 4: $103-110$

Whitelegge JP, Zhang H, Aguilera R, Taylor RM and Cramer WA (2002) Full unit coverage liquid chromatography electrospray ionization mass spectrometry (LCMS+) of an oligomeric membrane protein. Mol Cell Proteomics 1: 816-827

Whitmarsh J and Cramer WA (1978) Kinetics of the photoreduction of cytochrome $b-559$ by photosystem II in chloroplasts. Biochim Biophys Acta 460: 280-289

Widger WR, Cramer WA, Hermodson M, Meyer D and Gullifor M (1984a) Purification and partial amino acid sequence of the chloroplast cytochrome $b-559$. J Biol Chem 259: 3870-3876

Widger WR, Cramer WA, Herrmann RG and Trebst A (1984b) Sequence homology and structural similarity between cytochrome $b$ of mitochondrial complex III and the chloroplast $b_{6} f$ complex: position of the cytochrome $b$ hemes in the membrane. Proc Natl Acad Sci USA 81: 674-678

Widger WR, Cramer WA, Hermodson M and Herrmann RG (1985) Evidence for a hetero-oligomeric structure of the chloroplast cytochrome $b$-559. FEBS Lett 191: 186-190

Yan J and Cramer WA (2003) Functional insensitivity of the cytochrome $b_{6} f$ complex to structure changes of the hinge region of the Rieske iron-sulfur protein. J Biol Chem 278: 20925-20933

Zhang H, Huang D and Cramer WA (1999) Stoichiometrically bound $\beta$-carotene in the cytochrome $b_{6} f$ complex of oxygenic photosynthesis protects against oxygen damage. J Biol Chem 274: 1581-1587

Zhang H, Whitelegge JP and Cramer WA (2001) Flavonucleotide:ferredoxin reductase is a subunit of the plant cytochrome $b_{6} f$ complex. J Biol Chem 276: 38159-38165

Zhang H, Kurisu G, Smith JL and Cramer WA (2003) A defined protein-detergent-lipid complex for crystallization of integral membrane proteins. Proc Natl Acad Sci USA 100: 5160-5163

Zouni A, Witt HT, Kern J, Fromme P, Krauss N, Saenger W and Orth P (2001) Crystal structure of photosystem II from Synechococcus elongatus at $3.8 \AA$ resolution. Nature 409: 739-743 